\title{
Correction: Hematopoietic stem cell transplantation for children with acute myeloid leukemia-results of the AML SCT-BFM 2007 trial
}

Martin G. Sauer • Peter J. Lang • Michael H. Albert • Peter Bader • Ursula Creutzig • Matthias Eyrich • Johann Greil • Bernd Gruhn • Wolfgang Holter • Thomas Klingebiel • Bernhard Kremens · Heiko von der Leyen •

Christine Mauz-Körholz • Roland Meisel • Kirsten Mischke • Ingo Müller • Charlotte M. Niemeyer • Christina Peters (D) Christine Pohler • Dirk Reinhardt • Birgit Burkhardt • Paul G. Schlegel • Ansgar S. Schulz • Johanna Schrum •

Petr Sedlacek • Brigitte Strahm • Wilhelm Woessmann • Rupert Handgretinger • Martin Zimmermann •

Arndt Borkhardt iD

Published online: 25 November 2019

(c) The Author(s), under exclusive licence to Springer Nature Limited 2019

\section{Correction to: Leukemia}

https://doi.org/10.1038/s41375-019-0584-8
Since publication, author Bernd Gruhn noticed that their affiliation was incorrect. This has now been corrected in both PDF and HTML versions of the paper. 